\title{
An adaptive learning algorithm for spectrum sensing based on direction of arrival estimation in cognitive radio systems
}

\author{
Sala Surekha ${ }^{1}$, Md. Zia Ur Rahman ${ }^{1}$, Aimé Lay-Ekuakille ${ }^{2}$ \\ ${ }^{1}$ Department of Electronics and Communication Engineering, $K$ L University, Koneru Lakshmaiah Education Foundation, \\ Green Fields, Vaddeswaram, Guntur-522502, A.P., India \\ 2 Department of Innovation Engineering, University of Salento, Lecce, Italy
}

\begin{abstract}
In cognitive radio systems, estimating primary user direction of arrival (DOA) measurement is one of the key issues. In order to increase the probability detection multiple sensor antennas are used and they are analysed by using subspace-based technique. In this work, we considered wideband spectrum with sub channels and here each sub channel facilitated with a sensor for the estimation of DOA. In practical spectrum sensing process interference component also encounters in the sensing process. To avoid this interference level at output of receiver, we used an adaptive learning algorithm known as Normalised Least Absolute Mean Deviation (NLAMD) algorithm. Further to achieve better performance a bias compensator function is applied in weight coefficient updating process. Using this hybrid realization, the vacant spectrum can be sensed based on DOA estimation and number of vacant locations in each channel can be identified using maximum likelihood approach. In order to test at the diversified conditions different threshold parameters $0.1,0.5,1$ are analysed.
\end{abstract}

\section{Section: RESEARCH PAPER \\ Keywords: Adaptive learning; beam forming; cognitive radio; direction of arrival; spectrum sensing}

Citation: Sala Surekha, Md. Zia Ur Rahman, Aimé Lay-Ekuakille, An adaptive learning algorithm for spectrum sensing based on direction of arrival estimation in cognitive radio systems, Acta IMEKO, vol. 10, no. 4, article 13, December 2021, identifier: IMEKO-ACTA-10 (2021)-04-13

Section Editor: Francesco Lamonaca, University of Calabria, Italy

Received May 28, 2021; In final form December 1, 2021; Published December 2021

Copyright: This is an open-access article distributed under the terms of the Creative Commons Attribution 3.0 License, which permits unrestricted use, distribution, and reproduction in any medium, provided the original author and source are credited.

Corresponding author: Md. Zia Ur Rahman, e-mail: $\underline{\text { mdzr550gmail.com }}$

\section{INTRODUCTION}

Telecommunication systems are rapidly using from past few decades it leads to increase in frequency spectrum usage. Due to lack of frequency spectrum, there is low utilization of licensed and unlicensed bands. To avoid interferences, secondary users always must be aware of primary user is absent or present in a particular frequency band. Further considered secondary user direction of arrival (DOA), it contains multiple input single output (MISO) and one receiving antenna for primary user and feedback based adaptive frequency algorithm. Non orthogonal multiple access [1] based cognitive radio network is used for secure beamforming to avoid interference in multiple input multiple output networks. In array sensors, estimation of number of channels is a problem and it is solved by considering direction of arrival in spectrum sensing method. Wideband spectrum sensing channel [2] is divided into two sub channels; each sub channel is connected with sensor for processing and then estimation of DOA is done. For multi band signals, two spectrum scenarios are considered from sub-Nyquist samples: in first scenario, spectrum sensing method is examined then DOA is considered as recovery for frequency spectrum problems by proposing uniform linear array (ULA) ${ }^{[3]}$ with sensor at receiver then it is connected to equivalent circuit of analog front-end channel of modulated wideband converter (MWC). In second case, generalized likelihood ratio antenna beamforming [4] used for efficient and low complexity spectrum sensing. Localization technique is investigated in [5] depending on direction of arrival measurements for estimating primary users in cognitive radio networks. In ${ }^{[6]}$, [7] new spectrum sensing techniques based on beamforming are proposed. Null steering and joint beam-based resource allocation [8] used in femtocell networks in spectrum sensing. Performance of transmitter localization done using sector power measurements for every senso then derived Cramer Rao Bound (CRB) ${ }^{[9]},[10]$ for sector power estimation using DOA and mean square error is derived for analytical expression. Main objective of this paper, estimating DOA of various sensors for sensing [11], [12] the vacant spectrum and thereby facilitate channel allocation to secondary user. Here, we make use of an 
adaptive learning algorithm based on normalized least absolute mean deviation (NLAMD) strategy. In section 2, the DOA estimation with an adaptive leaning algorithm is discussed, in section 3 simulation results are discussed. The proposed realizations are suitable for the development of medical telemetry networks as well in the development of smart cities, smart hospitals.

\section{DOA ESTIMATION BASED SPECTRUM SENSING}

In cognitive radios, spectrum sensing is mostly used method because it overcomes the low spectrum utilization problems of primary users. There are various spectrum sensing algorithms they are based on narrowband methods used to solve binary hypothesis. This binary hypothesis test is used for every sub channel. For assessing the primary user's existence, sub channel assessed in spectrum sensing algorithm. In each sub channel received signal is expressed [13] as

$$
y_{k}=\left\{\begin{aligned}
w_{k}, & h_{0} \\
a r_{k}+w_{k}, & h_{1}
\end{aligned}\right.
$$

where $a r_{k}$ is primary user received signal, $w_{k}$ is additive white Gaussian noise, $h_{0}$ and $h_{1}$ are hypothesis test used for primary user existence in every sub channel. Multiple hypothesis tests are employed for spectrum sensing algorithm, then by taking into consideration of all sub channels primary user signal is detected. By analysing all sub channel received signals, observed that noise is present in output signal. To discriminate this noise from primary user signals, DOA is estimated. By assuming received signal at sensor nodes in array processing, it looks like cognitive radio subchannel, to avoid these problems DOA estimation is considered in spectrum sensing and its block diagram is shown in Figure 1. DOA estimation is considered to get exact information of antenna and also to avoid interferences between primary and secondary users, further an adaptive learning process called normalised least absolute mean deviation (NLAMD) algorithm is considered for spectrum to reduce noise levels at output. By using adaptive filter, desired response of input signal is calculated as

$$
d_{k}=s_{k}^{\mathrm{T}} u^{0}+o_{k},
$$

where $S_{k}$ is input signal, $\mathrm{u}^{0}$ is unknown weight vector with ' $\mathrm{t}$ ' taps and $o_{k}$ is output noise at time index ' $k$ '. Error output of desired signal is represented as

$$
e_{k}=d_{k}-s_{k}^{\mathrm{T}} u_{k} \text {. }
$$

Here, $u_{k}=\left[u_{k_{1}}, u_{k_{2}} \ldots ., u_{k_{T}}\right]^{\mathrm{T}}$ is weight vector of adaptive filter.

For above equation identification problem of adaptive system is solved by $\mathrm{p}$-norm cost function minimization

$$
J\left(e_{k}\right)=\frac{1}{p} \mathrm{E}\left[\left|e_{k}\right|^{p}\right]=\frac{1}{p} \mathrm{E}\left[\left|e_{k}=d_{k}-s_{k}^{\mathrm{T}} u_{k}\right|^{p}\right]
$$

where $E[$.$] is the operator of statistical expectation for \mathrm{p}>0$. $\mathrm{E}\left[\left|e_{k}\right|^{p}\right]$ is replaced with $\left|e_{k}\right|^{p}$, then after calculation we get gradient of $u_{k}$ for $\mathrm{p}$-norm error evaluated as

$$
\frac{\partial J\left(e_{k}\right)}{\partial\left(u_{k}\right)}=-\left|e_{k}\right|^{p-1} \operatorname{sign}\left[e_{k} s_{k}\right] .
$$

By using gradient descent algorithm weight equation is updated as

$$
u_{k+1}=u_{k}+\omega\left|e_{k}\right|^{p-1} \operatorname{sign}\left[e_{k} s_{k}\right],
$$

where ' $\omega$ ' is step size selected appropriately used for balancing convergence rate and mean square error, 'sign' used for denoting sign function. For improving steady state rate and convergence rate above equation is updated with normalised least mean $\mathrm{p}$ power algorithm as

$$
u_{k+1}=u_{k}+\omega \frac{\left|e_{k}\right|^{p-1} \operatorname{sign}\left[e_{k} s_{k}\right]}{\left\|s_{k}\right\|_{p}^{P}+\vartheta} .
$$

Here ||$.||_{\mathrm{p}}$ used for $\mathrm{p}$-norm operation, small positive value $\vartheta$ is also considered for avoiding denominator from zero. By selecting p-norm values as 1 , then we obtain the NLAMD algorithm equation as

$$
u_{k+1}=u_{k}+\omega \frac{\operatorname{sign}\left[e_{k} s_{k}\right]}{\left\|s_{k}\right\|_{1}^{1}+\vartheta} \text {. }
$$

In sparse models, $\mathrm{L}_{1}$ norm is used for relaxation in least absolute shrinkages and operator selector algorithms, and it is employed in various adaptive filter algorithms. By using $\mathrm{L}_{1}$ norm, weight equation of NLAMD algorithm is updated to minimise cost functions and it is given as

$$
J_{d}\left(e_{k}\right)=\frac{d_{k}-s_{k}^{\mathrm{T}} u_{k}}{\left\|s_{k}\right\|_{1}^{1}+\vartheta}+\varepsilon\left\|u_{k}\right\|_{1},
$$

where $\varepsilon$ is adopted parameter to know the difference between estimation error and sparsity. Then we get the updated equation for NLAMD sparse algorithm using gradient descent method using cost function equation (9) as

$$
u_{k+1}=u_{k}+\omega \frac{\operatorname{sign}\left(e_{k} s_{k}\right)}{\left\|s_{k}\right\|_{1}^{1}+\vartheta}-\sigma \operatorname{sign}\left(u_{k}\right) .
$$

Here, $\sigma=\omega \varepsilon$ is a regularised parameter. NLAMD algorithm with sparse system and $\mathrm{L}_{1}$ norm is denoted as ZA NLAMD algorithm.

In bias compensated systems [14], considered a noisy input system for NLAMD algorithm. Input noise vector of a system is defined as

$$
\bar{s}_{k}=s_{k}+o_{i n_{k}},
$$

where $o_{i n_{k}}$ noisy input vector it is represented as $o_{i n_{k}}=$ $\left[o_{i n 1_{k}}, o_{i n 2_{k}}, \ldots \ldots, o_{i n M_{k}}\right]^{\mathrm{T}}$, and its limit is $o_{i n, t_{k}}(l \in[1, M])$, their input variance is represented as $\sigma_{i n}^{2}$ and is estimated by using some unknown info. To recover the biased estimation problems for NLAMD algorithm of equation (10), an unbiased estimation vector $b_{k}$ is taken into consideration as

$$
u_{k+1}=u_{k}+\omega \frac{\operatorname{sign}\left(\hat{e}_{k} \hat{s}_{k}\right)}{\left\|\hat{s}_{k}\right\|_{1}^{1}+\vartheta}-\sigma \operatorname{sign}\left(u_{k}\right)+b_{k} .
$$

By using above equation, we get bias compensated vector as below [15]

$$
b_{k}=\omega \sigma_{\hat{e} \mid \hat{s}_{k}}^{2} \sqrt{2 /\left(\pi \sigma_{\hat{e} \mid \hat{s}_{k}}^{2}\right)}\left(\frac{u_{k}}{\left\|\hat{s}_{k}\right\|_{1}^{1}+\vartheta}\right) .
$$

Noisy input parameter variances $\sigma_{o_{i n}}^{2}, \sigma_{\hat{e} \mid \hat{s}_{k}}^{2}$ and $\sigma_{u_{k}}^{2}$ are estimated accurately by computing these variance parameters as 


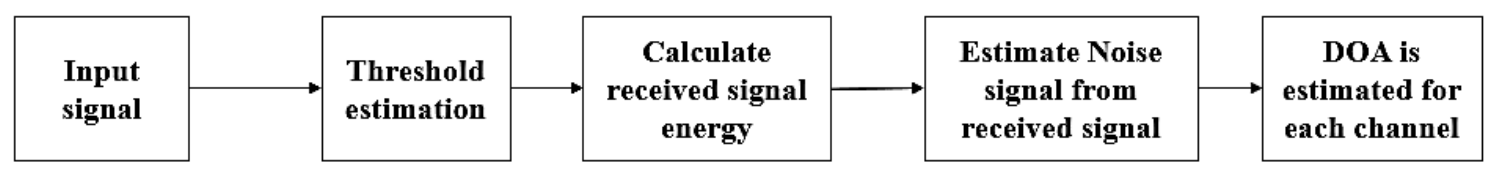

Figure 1. DOA estimation Block diagram for spectrum sensing.

$$
\begin{gathered}
\sigma_{o_{i n_{k}}}^{2}=\frac{\sigma_{\hat{e} \mid \hat{s}_{k}}^{2}}{M \sigma_{u_{k}}^{2}+i+\frac{\sigma_{\hat{e} \mid \hat{s}_{k}}^{2}(M)}{\hat{s}_{k}^{\mathrm{T}} \hat{s}_{k}}} . \\
\sigma_{\hat{e} \mid \hat{s}_{k}}^{2}=\aleph \sigma_{\hat{e} \mid \hat{s}_{k-1}}^{2}+(1-\aleph) \hat{e}_{k}^{2} \\
\sigma_{u_{k}}^{2}=\aleph \sigma_{u_{k-1}}^{2}+(1-\aleph) \frac{1}{M} u_{k}^{\mathrm{T}} u_{k} .
\end{gathered}
$$

Equation (13) is substituted into (12), we get final bias compensated NLAMD (BC-NLAMD) adaptative learning process updated as

$$
\begin{aligned}
& u_{k+1}=\left(1+\frac{\omega \sqrt{2 /\left(\pi \sigma_{\hat{e} \mid \hat{s}_{k}}^{2}\right)^{\sigma_{o_{i n}}^{2}}}}{\left\|\hat{s}_{k}\right\|_{1}^{1}+\vartheta} u_{k}\right. \\
& +\omega \frac{\operatorname{sign}\left(\hat{e}_{k} \hat{s}_{k}\right)}{\left\|\hat{s}_{k}\right\|_{1}^{1}+\vartheta}-\sigma \operatorname{sign}\left(u_{k}\right) .
\end{aligned}
$$

Using this weight recursion, the noise in the received signal is minimised and accurate direction of arrival is estimated. The BCNLAMD algorithm accurately estimates the DOA and helps in finding the vacant spectrum and the flowchart of the proposed adaptive learning algorithm is shown in Figure 2.

\section{RESULTS AND DISCUSSION}

This section demonstrates the experimental results for evaluating the performance of proposed bias compensated adaptive learning algorithm and is compared with absolute mean deviation (AMD) and normalised absolute mean deviation (NAMD) methods with output Gaussian noise. Input and output noises generated using zero white mean Gaussian noise and $\beta$ stable distribution respectively for better performance of proposed algorithm and its characteristic function is expressed as,

$$
f_{t}=\mathrm{e}^{j \delta_{t}-\varepsilon|k|^{\beta}\left[1+j \tau \operatorname{sign}(t) Q_{t, \beta}\right.},
$$

where

$$
Q_{t, \beta}=f(x)= \begin{cases}\tan \frac{\beta \pi}{2}, & \beta \neq 1 \\ \frac{2}{\pi} \log _{t}, & \beta=1\end{cases}
$$

with characteristic exponent $0<\beta \leq 2$, skewness $-1 \leq \tau \leq$ 1 , scale parameter range $0<\varepsilon<\infty$ and location parameter $-\infty<\delta<\infty$.

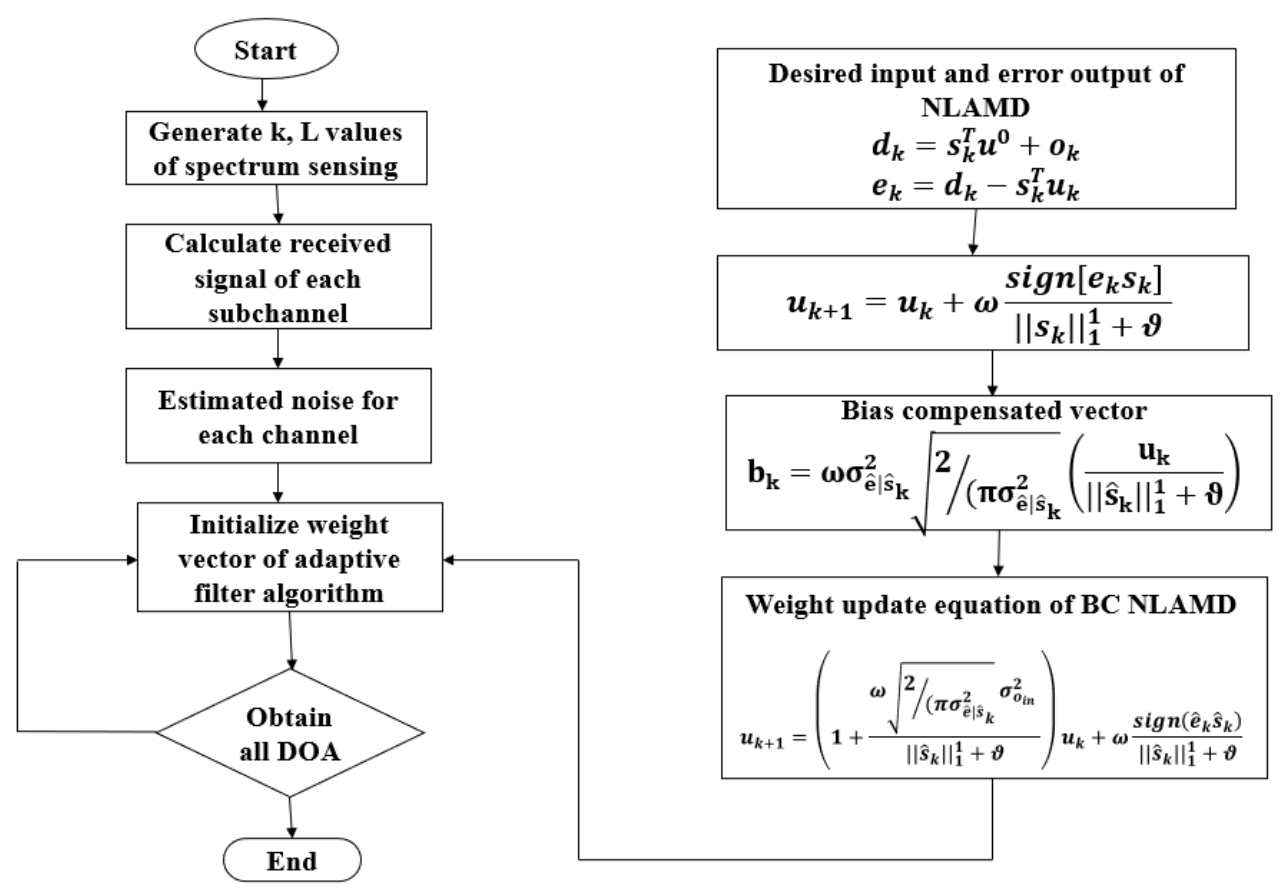

Figure 2. Flow chart of spectrum sensing DOA estimation using BC-NLAMD algorithm. 


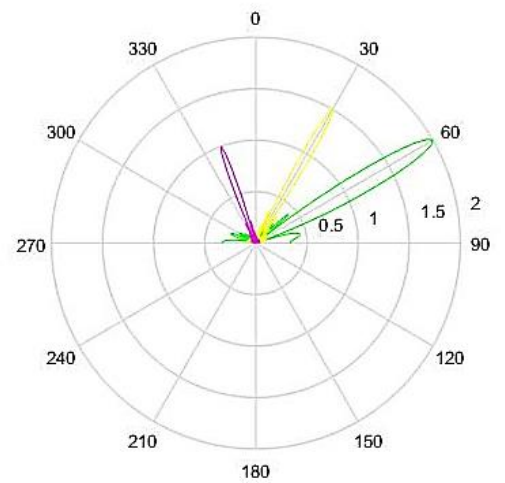

Figure 3. BCNLAMD Beam pattern for one white signal with DOAs.

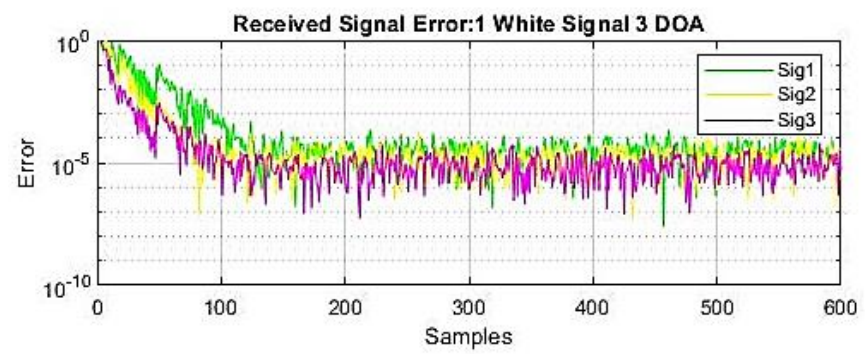

Figure 4. Error for received signal using BCNLAMD algorithm with threshold value of 0.1 .

Occupied subchannel locations are estimated using spectrum sensing method with choosing of Q subchannels. In cognitive radio applications, using spectrum sensing correlation between channels are identified. In our framework for identifying spectrum location direction of arrival is taken into consideration. By using DOA, we can identify spectrum location using the proposed BC-NLAMD adaptive learning process. Output signal with various combinations is considered as one white signal with one DOA, one white signal with $3 \mathrm{DOA}$, two white signals with one DOA and three DOA. Performance of adaptive algorithm is studied in terms of convergence rate, beam pattern and number of active taps. In mobile environments, more than one multipath is considered, in those each multipath have different gains and it has amplitude and phase components.

\section{Case 1: One white signal with one DOA}

In this type, one signal with one path is considered it arrives at base station with 60 degrees angle and amplitude 0.5 , it is propagated at different threshold values $0.1,0.5$ and 1 . For different threshold control values, delay and steady state error are calculated as discussed in second section. For threshold value of 0.1 , it improves convergence rate for proposed BC NLAMD algorithm when compared to LMS [16] algorithm. For threshold values of 0.5 and 1 steady state is converged faster than basic LMS algorithm. Improvement in convergence rate is identified by using taps in adaptive filter algorithm. For narrow band theoretical values, threshold value need only one tap but however it has delay cost and convergence rate problems. Hence, we considered beam pattern with NLAMD algorithm using MATLAB in DOA estimation, it steers main beam with 60 degrees direction with beam strength of two, it is due to power signal reduced by factor 0.5 . For every simulation, convergence rates are given in terms of number of samples, it is required for steady state and it is shown in Table 1.
Table 1. BCNLAMD Beam pattern for one white signal with DOAs.

\begin{tabular}{ccc}
\hline Algorithm & Steady state & Delay \\
\hline LAMD [17] & 65 & 0 \\
BBNLMS [18] & 50 & 0 \\
FFA [19] & 35 & 0 \\
PID [20] & 25 & 8 \\
ENLMS [21] & 15 & 12 \\
BCNLAMD for 0.1 & 40 & 0 \\
BCNLAMD for 0.5 & 45 & 7 \\
BCNLAMD for 1.0 & 55 & 14
\end{tabular}

\section{Case 2: One white signal with 3 DOAs}

These simulations are studied for effects of multipath smart antenna systems. Multipath antennas with three different direction of arrivals $-20,30$ and 60 degrees are considered for base station. At antenna system each multipath have a difference of one sampling period of $1 / f_{c}$ and their corresponding gains also introduced with amplitudes as shown in Figure 3. For proposed method, three different weight vectors for each multipath are used for spectrum sensing. Convergence rate for white signal with three DOAs gives better convergence rate for proposed bias compensated NLAMD algorithm when compared to AMD algorithm as shown in Figure 4. By proposed algorithm, antenna systems show similar beam pattern compared to basic AMD algorithm and it has ability to steer beams in multiple directions with zero interference directions for each beam, gain is inversely proportional to corresponding multipath of antenna.

\section{Case 3: Two white signals with one DOA}

Two different signals are transmitted with one DOA each and its effect is same as sending two multipaths for one signal separated by one sample period at least, it is due to two signals are uncorrelated for each other with amplitude of 0.5 and 1 at threshold values of $0.1,0.5$ and 1 . It gives good convergence rate for second signal when compared to first signal. For smaller amplitude it gives longer response to adapt taps and then signal is estimated. Narrow threshold gives an ability to adapt smaller number of taps for better performance of proposed NLAMD algorithm and their beam patterns for corresponding DOA are shown in Figure 5 and Figure 6 respectively.

\section{Case 4: Two white signals with three DOAs}

In this case, two input sequences are considered each sequence with three multipath components is simulated. At the base station, multipath signals of second and third are used behind first multipath signals from various directions. For each signal, only two sets of multipath are considered. At base station, second and third multipath have the same weight vector. Compared the convergence rate with basic LAD algorithm. Four beam patterns are considered from Shannon theory, $3^{\text {rd }}$ multipath pattern with two main lobes in direction of second and third multipaths, they are shown in Figure 7 and Figure 8 respectively. Convergence rates for first signal, second signal in case of DOA 3 is shown in Table 2 and Table 3 respectively in terms of number of samples considered to reach steady state. From the tables, it is clear that for DOA3 of proposed algorithm converged faster for second signal when compared to first signal. In Figure 7, only two multipath signals are visible because $2^{\text {nd }}$ and $3^{\text {rd }}$ signals have same weight vectors.

Main aim of proposed BC-NLAMD algorithm is for detecting frequency spectrums in cognitive radio antenna systems. NLAMD algorithm is used for low computational complexity, 


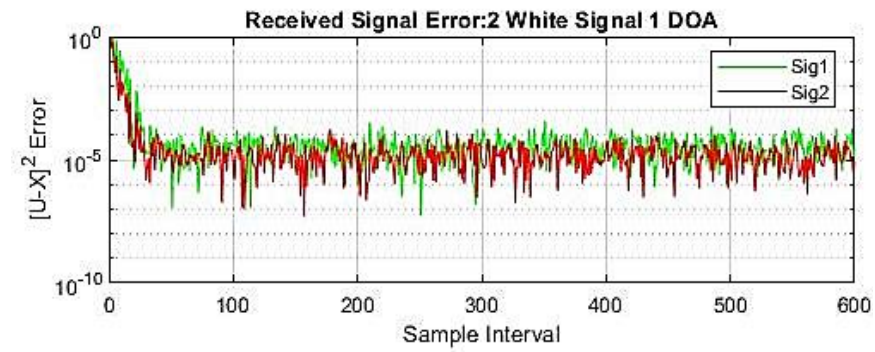

Figure 5. Error signal for BCNLAMD algorithm for 0.1 value.

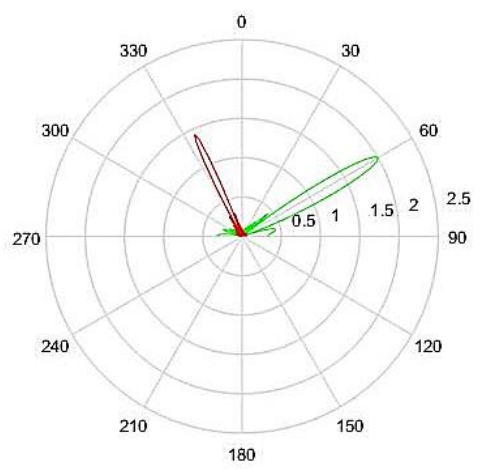

Figure 6. BCNLAMD Beam pattern for two white signals with one DOA.

better stability and good robustness to avoid implementation errors. However, NLAMD algorithm is poor convergence and it is increased by using bias compensated NLAMD algorithm and it requires system with sparse channels. In wireless channel, spatial properties are exploited with detection guide systems. Beam patterns are analysed and they are compared with Shannon theorem. Proposed results are compared with threshold point of spectrum sensing algorithm for BC-NLMAD algorithm. Then it gives better results for BC-NLMAD in terms of faster convergence, low computational complexity and particularly narrow band threshold gives better performance of antennas. It is due to delay period presence, on that time system waits for set of samples those are undetermined before actually converging for desired signal. However, system performance is improved at

Table 2. Convergence rate of first signal, DOA3.

\begin{tabular}{ccc}
\hline Algorithm & Steady state & Delay \\
\hline LAMD [17] & 55 & 0 \\
BBNLMS [18] & 60 & 20 \\
FFA [19] & 70 & 45 \\
PID [20] & 80 & 65 \\
ENLMS [21] & 90 & 70 \\
BCNLAMD for 0.1 & 35 & 60 \\
BCNLAMD for 0.5 & 55 & 40 \\
BCNLAMD for 1.0 & 125 & 95 \\
\hline
\end{tabular}

Table 3. Convergence rate of second signal and DOA3

\begin{tabular}{ccc}
\hline Algorithm & Steady state & Delay \\
\hline LAMD [17] & 45 & 0 \\
BBNLMS [18] & 55 & 0 \\
FFA [19] & 62 & 20 \\
PID [20] & 70 & 40 \\
ENLMS [21] & 85 & 55 \\
BCNLAMD for 0.1 & 25 & 35 \\
BCNLAMD for 0.5 & 45 & 25 \\
BCNLAMD for 1.0 & 95 & 65 \\
\hline
\end{tabular}
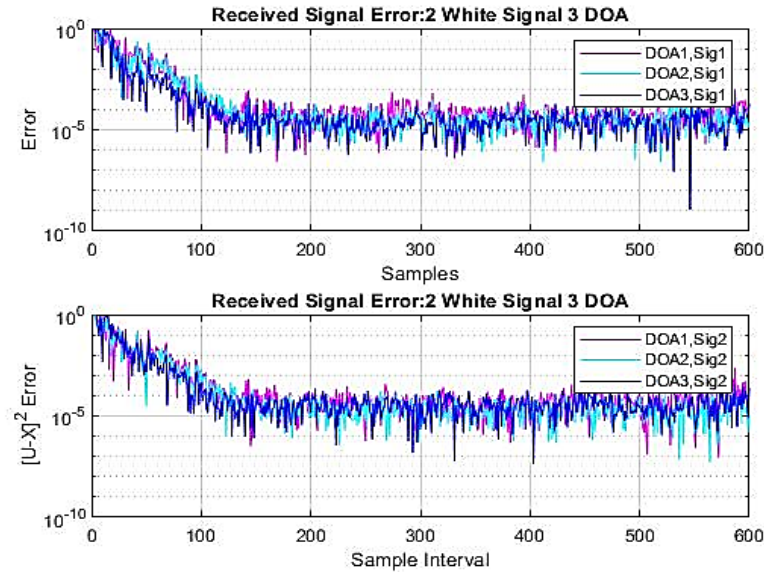

Figure 7. Received error signal for BCNLAMD algorithm.

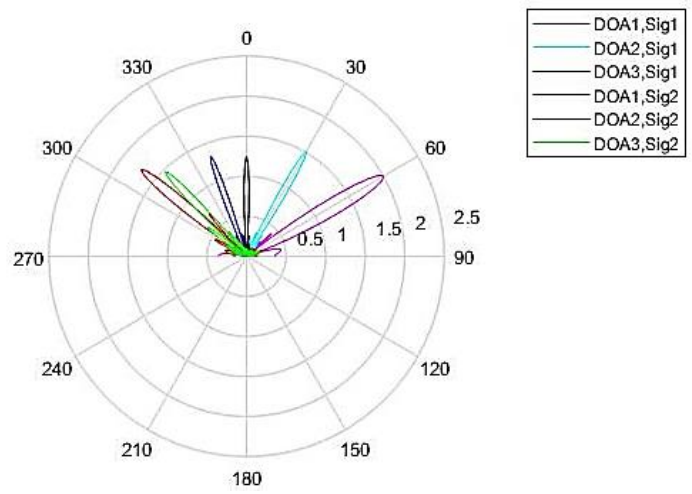

Figure 8. BCNLAMD beam pattern for two white signals with 3 DOAs.

a cost of increased computational complexity that will need increased number of taps adaptation for narrow threshold point in proposed algorithm and it reduces error at output signals. Beam patterns are obtained with according to expectations of Shannon theorem, and further proposed algorithm with active taps steers beams in desired signal direction. Spatial filtering is particularly used in wireless communication systems. Hence by proposed algorithm performance of system is increased with active taps weights in updated equation so that improves frequency utilization for cognitive radio systems.

\section{CONCLUSION}

In this paper, the vacant spectrum is sensed by using DOA measurement in wireless communication systems. Interferences occurred with cognitive radio systems are avoided by considering DOA estimation for spectrum sensing. In wireless communications, antenna system are new developing technologies with new adaptive beam forming algorithms, it will provide high frequency spectrum then it improves quality of service of cognitive radio systems. Further to reduce noise signals from received signal proposed a bias compensated NLAMD algorithm. Using this adaptive learning algorithm, performance of cognitive radio-based antenna beam streams and convergence rate is improved. By using sign regressor function in weight update equation of proposed algorithm computational complexity is reduced. Performance of BCNLAMD algorithm in presence of multipath users and multipath effects are analysed using MATLAB simulations and hence convergence rate is improved due to active taps used in adaptive learning algorithm leads to better spectrum efficiency in cognitive radios. 


\section{REFERENCES}

[1] H. S. M. Antony, T. Lakshmanan, Secure Beamforming in 5GBased Cognitive Radio Network, Symmetry, vol. 11, no. 10, October 2019, p. 1260.

DOI: $10.3390 /$ sym 11101260

[2] Amir Mahram, Mahrokh G. Shayesteh, Blind wideband spectrum sensing in cognitive radio networks based on direction of arrival estimation model and generalised autoregressive conditional heteroscedasticity noise modelling, IET Communications, vol.8, no.18, 2014, pp.3271-3279.

DOI: $10.1049 /$ iet-com. 2014.0162

[3] S. Stein Ioushua, O. Yair, D. Cohen, Y. C. Eldar, CaSCADE Compressed Carrier and DOA Estimation, IEEE Transactions on Signal Processing, vol. 65, no. 10, May 2017, pp. 2645-2658. DOI: $10.1109 /$ TSP.2017.2664054

[4] A. H. Hussein, H. S. Fouda, H. H. Abdullah, A. A. M. Khalaf, A Highly Efficient Spectrum Sensing Approach Based on Antenna Arrays Beamforming, IEEE Access, vol. 8, 2020, pp. 2518425197.

DOI: $10.1109 /$ ACCESS.2020.2969778

[5] J. Wang, J. Chen, D. Cabric, Cramer-Rao Bounds for Joint RSS/DoA-Based Primary-User Localization in Cognitive Radio Networks, IEEE Transactions on Wireless Communications, vol. 12, no. 3, March 2013, pp. 1363-1375. DOI: $10.1109 /$ TWC.2013.012513.120966

[6] H. S. Fouda, A. H. Hussein, M. A. Attia, Efficient GLRT/DOA spectrum sensing algorithm for single primary user detection in cognitive radio systems, International Journal of Electronics and Communications, 2018

DOI: 10.1016/i.aeue.2018.03.012

[7] S. Elaraby, H. Y. Soliman, H. M. Abdel-Atty, M. A. Mohamed, Joint 2D-DOA and Carrier Frequency Estimation Technique Using Nonlinear Kalman Filters for Cognitive Radio, IEEE Access, vol. 5, 2017, pp. 25097-25109.

DOI: $10.1109 /$ ACCESS.2017.2768221

A. Salman, I. M. Qureshi, S. Saleem, S. Saeed, B. R. Alyaei, Novel sensing and joint beam and null steering-based resource allocation for cross-tier interference mitigation in cognitive femtocell networks, Wireless Networks, vol. 24, no. 6, February 2017, pp. 2205-2219.

DOI: $10.1007 / \mathrm{s} 11276-017-1465-6$

[8] J. Werner, J. Wang, A. Hakkarainen, D. Cabric, M. Valkama, Performance and Cramer-Rao Bounds for DoA/RSS Estimation and Transmitter Localization Using Sectorized Antennas, IEEE Transactions on Vehicular Technology, vol. 65, no. 5, May 2016, pp. 3255-3270. DOI: $10.1109 /$ TVT.2015.2445317

[9] A. Lay-Ekuakille, P. Vergallo, D. Saracino, A. Trotta, Optimizing and post processing of a smart Beamformer for obstacle retrieval, IEEE Sensors Journal, vol.12, no.5, 2012, 1294-1299. DOI: $10.1109 /$ JSEN.2011.2169782

[10] M. A. Hussain Ansari, C. L. Law, Grating Lobe Suppression of Multicycle IR-UWB Collaborative Radar Sensor in Wireless
Sensor Network System, IEEE Sensors Letters, vol. 4, no. 1, Jan. 2020, Art no. 7000404, pp. 1-4. DOI: $10.1109 /$ LSENS.2020.2964588

[11] W. Lu, B. Deng, Q. Fang, X. Wen, S. Peng, Intelligent Reflecting Surface-Enhanced Target Detection in MIMO Radar, IEEE Sensors Letters, vol. 5, no. 2, February 2021, Art no. 7000304, pp. $1-4$. DOI: $10.1109 /$ LSENS.2021.3052753

[12] S. Surekha, M. Z. Ur Rahman, A. Lay-Ekuakille, A. Pietrosanto, M. A. Ugwiri, Energy Detection for Spectrum Sensing in Medical Telemetry Networks using Modified NLMS algorithm, 2020 IEEE International Instrumentation and Measurement Technology Conference (I2MTC), Dubrovnik, Croatia, 2020, pp. $1-5$.

DOI: 10.1109/I2MTC43012.2020.9129107

[13] Wentao Ma, Ning Li, Yuanhao Li, Jiandong Duan, Badong Chen, Sparse Normalized Least Mean Absolute Deviation Algorithm Based on Unbiasedness Criterion for System Identification with Noisy Input, IEEE Access, Vol.4, 2016, pp. 1-9.

DOI: $\underline{10.1109 / A C C E S S .2018 .2800278}$

[14] S. M. Jung, P. Park, Stabilization of a Bias-Compensated Normalized Least-Mean-Square Algorithm for Noisy Inputs, IEEE Transactions on Signal Processing, vol. 65, no. 11, 1 June 2017, pp. 2949-2961. DOI: $10.1109 /$ TSP.2017.2675865

[15] M. O. Bin Saeed, A. Zerguine, An Incremental Variable Step-Size LMS Algorithm for Adaptive Networks, IEEE Transactions on Circuits and Systems II: Express Briefs, vol. 67, no. 10, pp. 22642268, October 2020. DOI: $10.1109 /$ TCSII.2019.2953199

[16] V. C. Ramasami, Spatial Adaptive Interference Rejection: LMS and SMI Algorithms, Report, University of Kansas, April 2001

[17] V. A. Kumar, G. V. S. Karthik, A low complex adaptive algorithm for antenna beam steering, 2011 International Conference on Signal Processing, Communication, Computing and Networking Technologies, July 2011, pp. 317-321. DOI: $10.1109 /$ ICSCCN.2011.6024567

[18] M. L. M. Lakshmi, K. Rajkamal and S. V. A. V. Prasad, Amplitude Only Linear Array Synthesis with Desired Nulls Using Evolutionary Computing Technique, ACES Journal, vol.31, no.11, November 2016, pp.1357-1361. DOI: $10.1109 /$ WiSPNET.2017.8299890

[19] P. K. Mvemba, A. Lay-Ekuakille, S. Kidiamboko, An Embedded Beamformer for a PID-Based Trajectory Sensing for an autonomous Vehicle, Metrology and Measurement Systems, vol.25, no.3, 2018, pp.561-575. DOI: $10.24425 / 123891$

[20] K. Aravind Rao, K. Sai Raj, Rohan Kumar Jain, Implementation of Adaptive Beam Steering for Phased Array Antennas Using ENLMS Algorithm, Journal of Critical Reviews, vol.7, no.9, 2020 Doi: $\underline{10.31838 / \text { icr. } 07.09 .10}$ 